American Journal of Economics and Business Administration 2 (1): 120-128, 2010

ISSN 1945-5488

(C) 2010 Science Publications

\title{
Aspirin in Cardiovascular and Cerebrovascular Events: Does Market Failure Matter?
}

\author{
Roger Lee Mendoza \\ Cherry Hill, NJ 08003 USA
}

\begin{abstract}
Problem statement: Against the backdrop of the 2009 scientific studies qualifying the cardiovascular and cerebrovascular benefits of aspirin, two interrelated questions are raised for investigation in this study. First, why may the government intervene in an otherwise private transaction between physician and patient and between drug manufacturer and buyer, when it involves contentious pharmacological information? Second, does government intervention make a difference in what these transacting parties would otherwise have chosen to do in its absence? Approach: An Internet literature search was performed, using query term combinations, to identify relevant aspirin studies. The search yielded 61 juried publications that met our predetermined criteria for inclusion and thematic analysis. Results: Variance exists within the mix of economic and non-economic literature on aspirin information regulation. The study identified 4 instances of market failure that offer some of the most compelling theoretical and practical considerations for public policy intervention in the context of the 2009 findings. However, there is also indication that the sense of increased protection arising from safety regulations could stimulate risky behavior that nullifies their net protective effects or benefits. Conclusion: It is not clear either from the surveyed literature or existing economic theory if, ceteris paribus, regulated information alters or modifies the marginal propensity of a physician to recommend, and a patient to consume, aspirin to prevent cardiovascular and cerebrovascular events, particularly heart attacks, strokes and vascular death. The study suggests the need for policy reinforcements to safety information, if market failures are to be efficiently addressed and risk compensating behavior reduced.
\end{abstract}

Key words: Aspirin, cardiovascular, cerebrovascular, externalities, information, market failure, Pareto-optimal, principal-agent problems, prophylaxis, risk, risk compensation, Reye's syndrome, transaction costs

\section{INTRODUCTION}

Objectives and significance of the study: The question raised in the subtitle of this study is two-fold. First and in view of mainstream economists' opposition to drug regulation (Peltzman, 1973; Pearson and Shaw, 1994, Calfee, 1996; Klein, 2000), this study asked why governments can intervene in an otherwise private transaction between physician and patient and between drug manufacturer and buyer, when it involves contentious drug information. Second, this study attempted to determine from the existing literature if such intervention makes a difference in what these transacting parties would otherwise have decided or chosen in its absence.

Some form of pharmacological information regulation exists for aspirin in the United States and several countries. This study focuses on the anticipated costs and benefits of policy intervention in aspirin prophylaxis (preventive treatment) for cardiovascular (heart and circulatory system) and cerebrovascular (brain and blood vessels) issues. In doing so, it inquires into the potential effects of government regulation on the provision, allocation and utilization of scientific knowledge for cardiovascular and cerebrovascular events. This study also illuminates several health policy issues that countries, particularly in Asia and South America, have attempted to address in the wake of more recent (2009) findings about aspirin safety.

Evolution of aspirin's market value: Aspirin -- a pure and stable form of acetylsalicylic acid -- was discovered in the 1890s. By 1900, the pharmaceutical firm Bayer had obtained a patent to manufacture and market aspirin as a pain-relieving, fever-lowering and antiinflammatory tablet. Aspirin became available to the public without need for a physician's prescription by 1915 (Mann and Plummer, 1993). Further experimentation in the 1940s found that aspirin reduces the risks of a heart attack (damage to heart muscle due to lack of blood flow) and stroke (blocked artery which supplies blood to the brain). By the mid-1980s, it was scientifically accepted that aspirin works as an effective blood thinner to protect against platelet aggregation (blood clotting) that causes heart attacks and most strokes (Mann and Plummer, 1993). Other studies further suggested that Non-Steroidal Anti-Inflammatory Drugs (NSAID), like aspirin, hold significant promise in preventing several types of cancer by blocking 
cyclooxygenase enzymes. The human body produces these enzymes when inflammation occurs; they are equally produced by precancerous tissues (Thun et al., 2002).

Many physicians recommend aspirin prophylaxis to middle-age and older individuals for primary and secondary prevention. Physicians' off-label recommendations often come in the form of lower or "baby aspirin" doses of 81-150 mg. Aspirin as a form of primary prevention for healthy and low-risk patients is promoted, especially in the mass media, as an effective risk-reducer for heart attack, stroke and chronic problems, like hypertension and elevated cholesterol levels. Secondary prevention is geared towards high-risk individuals with cardiovascular and cerebrovascular history and to avoid the recurrence of another event (Lamotte et al., 2006a; 2006b). With cardiovascular and cerebrovascular diseases, respectively, as the first and third leading causes of death for American men and women (CDC, 2010), the prophylactic value of aspirin has consistently maintained or enhanced its strategic market value in the United States and other countries. One report, for example, noted that "Americans bought more than 44 million packages of low-dose aspirin marketed for heart protection in the year ended September (2009), up about 12\% from 2005" (Mathews, 2010).

Health risks and hazards: More recent research has raised questions about aspirin's scientifically validated health and medical benefits, especially for primary prevention. Prominent among these studies was one released in 2009 by an independent panel of health experts, known as the United States Preventive Services Task Force (USPSTF). Despite "good evidence that aspirin decreases the incidence of myocardial infarction (heart attack) in men and ischemic strokes (deceased area of brain tissue) in women," the report found that aspirin tends to deplete the stomach's protective lining. A depleted lining, in turn, increases the incidence of serious gastrointestinal bleeding (e.g., bleeding ulcers) and hemorrhagic strokes (strokes due to bleeding) (USPSTF, 2009). Some of these bleeding incidents could cause death. While aspirin is an effective inflammation-reducer for pain and fever by preventing prostaglandin production, the same report also found that this process could deplete a protective layer in the stomach and thus increase the risk of ulcer (USPSTF, 2009).

American federal and state public health agencies, led by the Centers for Disease Control and Prevention (CDC), issued official recommendations in 2009 and 2010. Thus far the closest that the United States government has approached regulation, the CDC focused specifically on a narrower group of patients who are at higher risk of a heart attack or stroke. The CDC guidelines came directly from the USPSTF report:

- Use of aspirin for men age 45-79 years, when the potential benefit due to a reduction in myocardial infarctions outweighs the potential harm due to an increase in gastrointestinal hemorrhage

- Use of aspirin for women age 55-79 years when the potential benefit of a reduction in ischemic strokes outweighs the potential harm of an increase in gastrointestinal hemorrhage

- Current evidence is insufficient to assess the balance of benefits and harms of aspirin for cardiovascular disease prevention in both men and women 80 years or older

- Avoid use of aspirin for stroke prevention in women younger than 55 years and for myocardial infarction prevention in men younger than 45 years

The cost-calculus: Several studies that predated the 2009 USPSTF report, including one that the same task force had completed in 2002, were broader in scope and less specific in terms of clinical guidelines. The controversy and disagreement within the medical and scientific communities, spawned largely by the 2009 USPSTF report, stemmed from the USPSTF's proposed cost calculus. This differentiated risk levels among individuals based on their demographic characteristics more than their physical or health condition. The demographic factors included age, gender and weight.

At bottom lay two chief concerns about the suggested cost calculus: (1) How should a decisionmaker (physician, patient and government agency) strike an objective balance between aspirin's potential health side effects and therapeutic benefits when net benefits are disputed? (2) Which factors should be assigned greater weight in assessing aspirin's negative effects in cardiovascular and cerebrovascular prevention?

On these grounds, certain key findings of the USPSTF report have been questioned and qualified by a stream of studies that were published that same year. Foremost among these was a meta-analysis of serious vascular events conducted by an international team of scientists using practically the same data from the 2009 USPSTF report. Although they conceded the substantial net benefit of low-dose aspirin for people with occlusive vascular disease, this team, led by the Antithrombotic Trialists' Collaboration (ATT), disagreed that age was the primary risk determinant. Chronic illnesses, like diabetes and high blood pressure, were cited as equally significant determinants of an individual's risk to internal bleeding (ATT Collaboration et al., 2009). Another major source of disagreement concerned the USPSTF finding about the 
varying effects of aspirin on men and women and the gender-based recommendations about heart attacks and ischemic strokes. The ATT-led team has disputed these findings and concluded that "[i]n both primary and secondary prevention trials, the proportional reductions in the aggregate of all serious vascular events seemed similar for men and women" (ATT Collaboration et al., 2009). ATT Collaboration et al. (2009) expressed further reservations about the significance of baseline risk. A rebuttal from the USPSTF asserted that gender and baseline risk matter significantly when considering net therapeutic benefits (Calonge and LeFevre, 2009), as it referenced another scientific finding (Algra and Greving, 2009).

The ATT-led study derived some empirical support from another published study in 2009. Its authors concluded that aspirin prevents heart attacks and ischemic strokes but also heightens risks of hemorrhagic strokes and other serious bleeding events in primary prevention for men and women (Wolff et al., 2009). Creating further confusion among many aspirin consumers was a late 2009 study that found insignificant difference in the net cardiovascular benefits of aspirin to patients with and without diabetes (Calvin et al., 2009).

In the absence of clear guidance as to whether aspirin's benefits for primary prevention are outweighed by the risks of its side effects, many patients and doctors have been left searching for their own position, some on the basis of non-medical considerations (Mathews, 2010). This has prompted the claim of some scientists that the net result of any costcalculus would eventually have to depend largely on their personal judgment (Dominiczak, 2009). The United States has opted to regulate indirectly and informally through the CDC guidelines. Other countries in Asia and South America have responded to the ensuing scientific stalemate by establishing safety regulations to pharmacological information and drug advertising for aspirin.

We proceed at this point to theoretically examine the rationale and efficacy of public policy intervention in the provision, allocation and utilization of aspirin pharmacological information.

\section{MATERIALS AND METHODS}

An Internet literature search of juried studies about aspirin and NSAIDs was undertaken using query term combinations of "aspirin" and the following: "regulation," "prevention" and "risk compensation" (including terminology variants). The net yield was 61 separate studies, after the Internet list was purged of duplicates, opinion-journalism pieces and reviews. These studies were thematically, rather than quantitatively, analyzed for insights into the behavior of aspirin decision-makers relative to its costs and benefits. The possible role and extent of government intervention in the prevention of vascular events were also gathered from the surveyed literature as well as prevailing economic scholarship.

\section{RESULTS}

Based on our thematic analysis of 61 juried publications, we identified 4 separate sources of market failure. These generally appear to support the case for mandatory disclosure of safety-related information, given that the net benefit of aspirin prophylaxis, particularly in primary prevention, is disputed.

From a traditional economic standpoint, market failure derives from individuals' pursuit of rational selfinterest that leads to Pareto-inefficient results. Another outcome exists where the overall gains from the new outcome outweigh individual losses, even considering that some individuals may lose under the new arrangement (Pindyck and Rubinfeld, 1989). From a political standpoint, market failure exemplifies situations or conditions where market forces and transactions do not serve, or run counter to, the public interest (Buchanan, 1988; Brennan, 2008). These economic and political approaches were analytically applied to the 4 sources of market failure discussed below.

Externalities: As an over-the-counter drug, the decision to take aspirin to prevent a heart attack, stroke and other vascular events ultimately rests upon the patient (Keith, 1995). The private costs of aspirin side effects, arising from its actual harms, are relatively high. They include medical and hospitalization expenses, absenteeism from employment, early retirement, income loss, disability or even premature death.

Personal costs impose negative spillover effects on third parties that include the individual's family or household (quasi-externalities) and other people and society as a whole (externalities). To household members, direct consumption and savings costs result from losses in the affected individual's income, employment and savings opportunities (particularly if s/he is an income earner). Returns on investments (e.g., based on costs borne by parents and other family members for the affected individual's health, education and welfare) diminish when one suffers from the serious side effects of aspirin Care of an individual by family members further entail direct expenditures of their personal time, effort and attention as well as psychological costs (pain, anxiety, bereavement and adjustments to a patient's behavioral changes). The 
external effects on non-family members exist in a variety of forms (e.g., impact of an individual's reduced work performance to the firm and co-workers). Societal costs include both direct costs (e.g., state disability and unemployment insurance) and indirect costs (e.g., loss of a vital source of labor supply and productivity and impact on mortality rates). Conversely, the therapeutic benefits of aspirin prophylaxis to a patient create positive externalities and quasi-externalities.

Despite these external consequences, various economic studies suggest that government regulations tend to induce compensating (or offsetting) behavior on the part of consumers of products that pose health risks. Risk compensation refers to behavioral responses or outcomes whereby the additional safety offered by regulation is "used up" or offset through more risky actions. These result from an individual's increased sense of protection. The offsetting behavior, in turn, creates or increases other externalities (positive or negative) when regulations are engineered to reduce the typical loss suffered by individuals per accident or "loss event" (Traynor, 2003). For example, if some health benefits predicted from information regulation (e.g., onlabel and advertisement warning of gastrointestinal bleeding or hemorrhagic stroke) become performance improvements, positive externalities to society result from compensatory behavior. On the other hand, negative externalities can obtain from a higher economic utility gained by an individual from not reading or heeding drug safety information.

Another study indicates that certain factors motivate or influence risk compensation depending on their level of intensity. These factors are visibility, effect, motivation and control (Hedlund, 2000). Hence, if the aspirin warning is highly visible, affects the patient substantially, offers good reasons or motivations to change consumption or user behavior and gives the patient the freedom or opportunity to do so, offsetting behavior is likely. At the same time, the expected higher likelihood of risky compensation from safety and advertisement warnings reveals two critical shortcomings: (1) Any economic analysis is purely theoretical because risk simulation and other experimental evaluations are "contaminated with poor data and uncontrolled factors" and, therefore, "cannot provide useful evidence" (Hedlund, 2000); (2) It is not clear "whether the overall (behavioral) effect will be to compensate partially, completely, or more than completely for the safety measure" (Hedlund, 2000).

Principal-agent problems: Related to, but distinct from, the issue of external effect is the principal-agent problem. Actions of agents, like doctors and pharmaceutical (and their research) firms, can have externalities that are innate to the methods of production, or other conditions important to the market. To illustrate, when a physician sees a patient and recommends aspirin for primary or secondary prevention, the physician must naturally pay for his/her professional time and expertise, staff, office/clinic facilities and other inputs which are reflected in the insurance portion of medical fees and patient copayments.

However, agency problems abound. One study suggests that American physicians are less likely than their European counterparts to counsel and discuss behavioral interventions with their patients (e.g., lowering blood pressure numbers through diet and lifestyle changes). Instead, American physicians tend to rely on prescribing medications (McKinlay et al., 2006) like aspirin. In doing so, risk compensation theory suggests that one set of clinical guidelines for primary prevention (USPSTF, 2009) could be overvalued by physicians, even if they may be more costly than others that do not put a premium on age, gender or other baseline factors (ATT Collaboration et al., 2009). In other countries like Japan, where physicians typically prescribe and dispense drugs, a study has shown that the markup influences and distorts physicians' prescription decisions. Some Japanese patients could thus take prophylactic drugs when none is necessary, while the liability of the doctor-agent is either unrecognized or unenforced (Iizuka, 2006).

In short, if aspirin prophylaxis leads to serious gastrointestinal bleeding, hemorrhagic strokes, cardiovascular death and other medical complications, neither the recommending physician nor the drug maker generally bear any compensatory burden. Private law failures (more than market failures) explain why these agents are relatively insulated from regulation liability: "the insolvency risk, the difficulty for the average patient in recognizing the etiological relation between harm and its cause..., the presence of causal uncertainty,... and given that a long length of time often elapses before the harm manifests itself" (Arcuri, 1999). Instead, whatever the magnitude of the direct and spillover costs may be has to be borne by the patient and third parties, such as insurers, governments and society in general. The market price for aspirin will fail to incorporate the full opportunity cost of producing it (i.e., Pareto-sub optimal allocation or market equilibrium will prevail). More aspirin will be produced and marketed than would occur were its recommending physicians, manufacturers and researchers paid for its associated costs or negative consequences. The marginal social cost of aspirin will therefore exceed its marginal social benefit. Because aspirin production and allocation (including advertising and marketing to target population groups) cannot efficiently take externalities into consideration and private law cannot 
cover these types of risks, a justification exists for some form of information regulation.

Our literature search generated 4 studies, which imply that the "risk thermostats" of agents have to be reset by offering incentives capable of motivating them to alter the amount of risk they are willing to incur for their principals (Wilde, 1994). Pharmaceutical compliance with disclosing hemorrhagic risks, or a government-sponsored educational campaign about harms, may not reduce the propensity of doctors to routinely recommend aspirin or patients to buy it. Target risk (the level of risk one chooses to accept in order to maximize the overall expected benefit from a decision or activity) may not therefore change on the part of doctors, drug makers or even patients unless safe behavior is rewarded and riskier choices contain liabilities (Wilde, 1994). A carrot-and-stick approach might involve, among others, the creation of medical review panels for aspirin prophylaxis recommendations, lower health and life insurance premiums for patients who do not suffer from internal bleeding.

Information constraints: Information can be considered a public good in an economic and political sense. Economically, it is non-excludable and non-rival. On the other hand, its provision by government serves a public interest or equity purpose because market forces do not operate well to encourage and support the production of knowledge.

Two types of information constraints may develop in the absence of direct or indirect policy intervention in aspirin prophylaxis. Asymmetric information occurs because one party to a transaction (pharmaceutical firm) has an incentive not to disclose information about aspirin's harms and risks that can negatively affect its aggregate level of market demand. Yet, because a key part of a pharmaceutical firm's operation is the production of drug information (e.g., through scientific research), the information it possesses and at its disposal tends to be better or more than what a patient or his/her family is able to gather or validate. Imperfect information is information that is not deliberately concealed by one transacting party from the other. Rather, it exists because of inadequate access and processing capabilities on the part of the aspirin consumer. In the case of aspirin, the current debate concerning which age and weight groups and gender are at higher risk exemplifies the long-time lag between consumption behavior and the outcome of behavioral change. Insufficiently understood, accepted or communicated health side effects owes in large part to the incremental processes of scientific discovery, information dissemination and peer acceptance (Mendoza, 2008). In the meantime, this long-time lag may take its heavy toll on non-risk patients whose private marginal consumption costs are higher than their private marginal benefits from primary prevention. Lack of good information about risks frequently occurs in many countries, particularly in the developing world, as a result of the scarcity and limited dispersion of resources and lack of competitive prowess of the poor and marginalized populations.

Until the 1990s, when both baseline and aspirincaused increases in risks and harms were deemed small, economists were generally critical of the United States Food and Drug Administration (FDA) policy of restricting information about aspirin's cardiovascular benefits (Keith, 1995). With knowledge and acceptance of its therapeutic effectiveness now widespread and scientific studies underscoring its harms in primary prevention growing, risk information is seen by some surveyed studies as vital in objective decision-making. If government regulation forces drug suppliers to reveal information about aspirin risks, on which basis people can presumably make better choices, market transactions will ensure that preferences are met. Mandatory disclosure will also reduce costs where the aspirin user is the least cost-abater. In addition, lowdose users would be encouraged to take a more discriminating view toward visible on-label and advertisement safety guarantees (e.g., "enteric-coated aspirin for added stomach protection," "protects your heart by keeping blood flowing freely," "often recommended as baby aspirin by doctors for adults"). The costs of formulating and enforcing disclosure rules are quite low.

Yet, allocation problems exist among users of drug information. For one, there are "information digestion" issues. The target user might be unwilling to read or unable to fully comprehend the warnings about harms and risks while some safety guarantees can be misleading (Ogus, 2001). There are also distributional consequences of information remedies, which tend to favor the "relatively well-off" (Pildes and Sunstein, 1995) and which underscore the need for public education (Arcuri, 1999). "Bounded rationality" theory, on the other hand, offers evidence that target users "tend to overestimate risks associated with lowprobability events and underestimate those arising from higher-probability events" (Ogus, 2001).

Because the costs of consumer error in assimilating pharmacological information and making decisions based on such information, are high when they involve serious personal injury or loss of life, it is not very clear either from economic theory or our reviewed literature if the benefits of government-mandated disclosure outweigh its costs from the perspective of the decisionmakers. The lack of medical and scientific consensus over the net benefit of aspirin prophylaxis for primary 
prevention further complicates the consumer's own cost-calculus.

Coordination and transaction costs: Transaction costs appear to offer another strong case for limited policy intervention in drug information provision. This is mainly because the costs of coordination are so high that it is usually cheaper for government to exercise its regulatory powers to prescribe conduct, such as in the case of aspirin prophylaxis.

Information search costs tend to be high because inconclusive, incremental, confusing or conflicting scientific criteria and findings make it challenging for physicians and patients to establish if the latter will most likely be better or worse off taking aspirin. A Coasean bargain would not typically obtain in the case of aspirin prophylaxis, even if well-defined property rights and rational actions exist. This is because it is difficult, if not impossible, for an aspirin consumer to negotiate directly with the drug maker or advertiser (e.g., to provide product warnings or conduct further research on harms and risks). Just as in the case of producing a pure public good, the problems of negotiating a unanimous contract become larger the larger the number of people or patients involved. Organizing patients for bargaining purposes would then become practically indistinguishable from government action (e.g., class action and product liability suits in tort law). Finally, assuming that a large group of people succeeds in compelling drug manufacturers and advertisers to give in to their bargaining demands, contractual enforcement and compliance monitoring on the part of these people would be costly, besides the problems with organizing again to achieve their transaction objectives.

Since transaction costs are jointly produced by the actions of transacting parties, it goes without saying that aspirin side effects would only arise if there were aspirin-recommending doctors or aspirin-buying patients, despite its mass production, aggressive marketing and over-the-counter availability. How costly is it to the government to efficiently reduce transaction costs through coordinated interventions (e.g., drug literature regulation, policing misleading information, public education) and whether the costs of corrective action do not exceed its social benefits, have not been sufficiently established and require empirical investigation.

One study indicates that cardiovascular secondary prevention involving aspirin use, when combined with controlling pre-diabetes, weight reduction among obese or near-obese individuals, lowering blood pressure for diabetic individuals and lowering LDL cholesterol for those with existing coronary artery disease, offers the greatest positive externalities to the American population. But since most of these prevention activities are also expensive, both at current prices and as currently delivered, reducing their cost is of critical importance (Kahn et al., 2008).

Finally, some of our surveyed studies cast doubts on whether a patient's economic utility (e.g., in terms of exercising greater caution, seeking a second medical opinion, comparing experiences with other patients) is improved by lowering the transaction costs of enforcing corporate responsibility and compliance. In this regard, it is well to remember that desired outcomes could theoretically be achieved by private transactions among the concerned parties were it not for the high cost of coordination. Whether risk compensation contains a reverse reciprocal effect on transaction cost reductions from information regulation awaits further investigation.

\section{DISCUSSION}

Market failure is often a controversial subject in economic theory (Cowen, 1988). It appears to generate greater concerns when government intervention has to assess the risk probabilities of pharmaceutical products using science. The processes of scientific discovery and peer acceptance, as the aspirin issue demonstrates, are often slow and incremental. As the body of scientific evidence grows, so does the propensity of scientists to further investigate and test its validity (Mendoza, 2008). Depending on how scientific evidence is used or applied (e.g., to establish clinical guidelines for aspirin use), risk valuations could also be inconclusive or incomplete.

Despite its inherent limits, some would assert that scientific expertise ought to be fully integrated into regulatory decision-making (Hankin, 1996). As one legal scholar succinctly put it, "[s]cience is the only method for ranking risk. In addition, it should be remembered that science is knowledge so systematized that prediction and verification are still possible, which implies that the public can monitor the work of regulators" (Arcuri, 1999). This underlines the role of drug safety regulation in determining which risks, based on existing scientific evidence, it should accept and address. For this reason, market failure justifications tend to eventually move away "from the determination of risks to the decisions concerning risk" (Arcuri, 1999).

Risk-based decisions ultimately bear on government performance and accountability in addressing market failures. One decision that aspirin regulators will have to make involves the weight it will assign to certain risks that have been identified by the medical and scientific communities (e.g., whether to give more credence to the USPSTF or ATT-led study). 
Addressing market failures will proceed from that decision. For example, public policy interventions can choose to correct the imbalanced or inadequate flow of information without necessarily imposing costs on other people and society in general that would exceed the social benefits of such corrective action. Establishing mechanisms by which the party with better or more information (e.g., pharmaceutical and research firms) can share valuable information, or by which the former's market power is discouraged from distorting the information it shares with aspirin buyers, could take various forms. As our literature search has indicated, these may include mandatory risk labeling, marketing and advertising monitoring, regulatory guidelines such as those issued by the CDC and state health agencies, medical peer review committees and support for scientific research and discussion. Public education and information dissemination campaigns should take into consideration certain disadvantaged groups that are more likely to be less informed.

Reducing the information deficit alone for aspirin buyers does not appear to directly promote change in consumption behavior. Rather, it signals a broader perspective of market failure that incorporates some social and political justifications. The primary purpose of information regulation in this instance is to make the accessibility and distribution of safety information more equitable in an otherwise unregulated market. The equity concept raised by this study involves not only mandating risk information, but also addressing misleading benefit information. The scientifically disputed claim of drug makers and advertisers that enteric-coating reduces the risk of significant stomach bleeding or makes aspirin "safer" (UC Berkeley, 2006) exemplifies the need for a limited form of information regulation, whether or not it changes the marginal propensity to recommend or purchase aspirin.

On-label and literature warnings of Reye's syndrome exemplify the delimited regulation that may be appropriate for the potential costs and benefits of aspirin prophylaxis. Reye's syndrome -- a rare but deadly disease that causes damage to the brain, liver kidney and other vital organs -- has been associated with aspirin consumption by children. However, scientific evidence is no less disputed, since it occurs among children with viral illness who do not take aspirin. FDA recommendations concerning the consumption of aspirin and other aspirin-containing drugs by children nonetheless constitute mandatory product safety information.

Public policy intervention could also stimulate incentives for doctors and patients to take the third party impacts of their aspirin decisions into account if it accepts certain risks (such as bleeding ulcers, hemorrhagic strokes or vascular death) as serious.
Besides implying that the competitive equilibrium will not result in the social optimum for aspirin consumption, externalities suggest that the same equilibrium will result in a dead weight loss. People who would have more marginal costs than marginal benefits, by virtue of their demographic and health characteristics, will be more inclined to buy aspirin to contain cardiovascular, cerebrovascular, oncological and other health issues.

Figure 1 shows the aspirin market before risk information is mandated (or questionable safety claims prohibited), with aspirin quantity supplied, Q1, at market price, $\mathrm{P} 1$.

After mandatory information on side effects is added, the quantity of aspirin supplied is reduced in Fig. 2, as Q1 moves away from market equilibrium to Q2. The dead weight loss resulting from information regulation is denoted by the triangular area B. It should be noted that the smaller the elasticity of supply and demand, the smaller the actual dead weight loss. Areas $\mathrm{A}$ and $\mathrm{B}$ represent losses in aspirin producer and consumer surpluses.

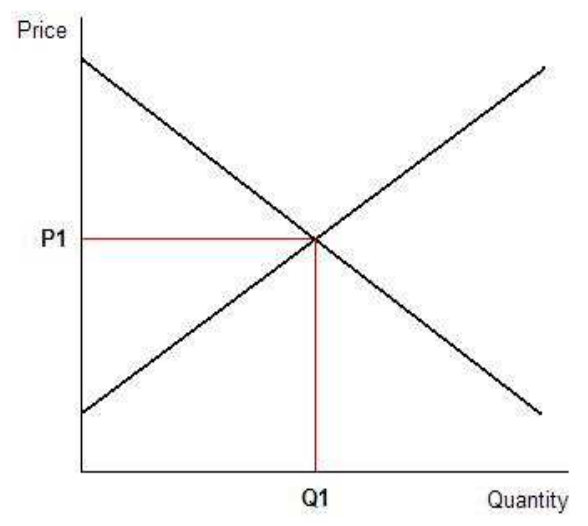

Fig. 1: Aspirin demand before safety information

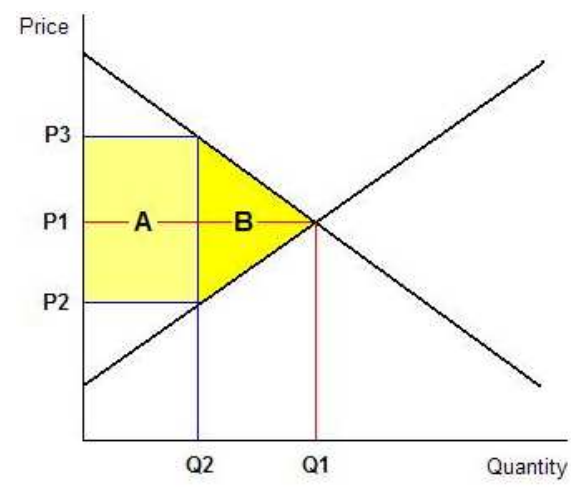

Fig. 2: Aspirin demand (with dead weight loss) after new regulation 


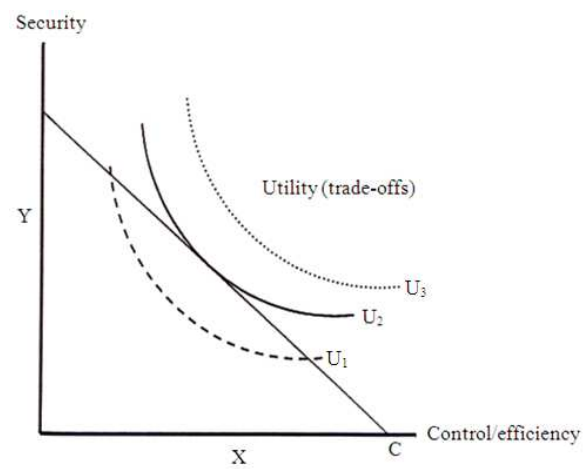

Fig. 3: Risk compensation in secondary prevention

By opting for limited regulation along the lines of the Reye's syndrome warning, a government makes the conscious paternalistic decision to objectify -- not supplant -- individual judgment where the net benefits of physician-recommended primary treatment or drug maker-claimed safety may are inconclusive or unvalidated. To a certain extent, the same paternalistic (or non-economic) rationale could be asserted for secondary prevention of cardiovascular and cerebrovascular diseases. Without legitimate safety provisions built into drug information, under-utilization and non-compliance by high-risk patients could be as much of a problem as it may be for those who are induced to take aspirin for what the ATT deems to be inappropriate reasons (i.e., for primary prevention). The risks of gastrointestinal bleeding, hemorrhagic strokes and other complications could be magnified (e.g., in the mass media) to the point where offsetting behavior among high-risk patients is encouraged.

Figure 3 depicts the trade-offs between the relative security offered by secondary prevention, Y and some hypothetical benefit/s (control and efficiency of alternative) obtained by some high-risk patients from compensating behavior, X. Given the level of security and the level of preference divergence depending on a patient's health condition, line $\mathrm{C}$ denotes the securityrisk compensation trade-offs. These trade-offs offer a series of indifference curves, $\mathrm{U}_{1}, \mathrm{U}_{2}$ and $\mathrm{U}_{3}$, with $\mathrm{U}_{3}$ having the greatest utility to the high-risk patient/s. In this sense, non-economic justifications for government intervention in pharmaceutical market failures contain both symbolic and practical value.

\section{CONCLUSION}

Externalities, agency problems, asymmetric and imperfect information, coordination and transaction costs offer compelling arguments in favor of on-label and advertising information regulation for aspirin prophylaxis. These considerations are both economic and non-economic in nature, which perhaps suggest some divergence between mainstream economic thought and medical/scientific views about the efficacy of some form of safety provision. In the same way that economists generally oppose regulation restricting drug benefit information due to its potential risks, this study finds that scientific information about harms cannot be ignored or underestimated even when a drug's therapeutic effects are substantial.

However, it is not clear from the surveyed literature mix if, ceteris paribus, regulating on-label and advertising information directly encourages or alters aspirin decisions for the prevention of heart attacks, strokes and other vascular events. Mainstream economic research point to a myriad of demographic and health factors that affect the choices that physicians and patients make concerning on-label and off-label aspirin utilization. Risk compensation has also been identified in this study as an important feedback effect that could reduce or nullify any protection offered by information regulation. Therefore, the challenge for governments is to seek fair representation of health cost and benefit information and ensure its availability to aspirin decision-makers.

\section{ACKNOWLEDGMENT}

The author wishes to acknowledge with thanks the helpful comments and suggestions made by his faculty colleagues and this journal's anonymous peer reviewers in improving the various drafts of this paper.

\section{REFERENCES}

Algra, A. and J.P. Greving, 2009. Aspirin in primary prevention: sex and baseline risk matter. The Lancet, 373: 1821-1822. DOI: 10.1016/S01406736(09)60503-1

Antithrombotic Trialists' (ATT) Collaboration, C. Baigent, L. Blackwell, R. Collins and J. Emberson et al., 2009. Aspirin in the primary and secondary prevention of vascular disease: collaborative metaanalysis of individual participant data from randomized trials. The Lancet, 373 (9678): 18491860. DOI: 10.1016/S0140-6736(09)60503-1

Arcuri, A., 1999. Product Safety Regulation. In: Encyclopedia of Law and Economics, Bouckaert, B. and G. De Geest, (Eds.). Edward Elgar and the University of Ghent, Ghent, ISBN: 185898565X, pp: 329-344.

Brennan, G., 2008. Market failure: Compared to what. Eth. Econ., 6: 1-6.

Buchanan, J., 1988. Market failure and political failure. Cato J., 8: 1-13.

Calfee, J.E., 1996. The Leverage Principle in the FDA's Regulation of Information. In: Competitive Strategies in the Pharmaceutical Industry, Helms, R.B. (Ed.). American Enterprise Institute, Washington DC., ISBN: 10: 084473882, pp: 302-321. 
Calvin, A.D., N.R. Aggarwal, M.H. Murad, Q. Shi and M.B. Elamin et al., 2009. Aspirin for the primary prevention of cardiovascular events. Diabetes Care, 32: 2300-2306. DOI: 10.2337/dc09-1297

Calonge, N. and M. LeFevre, 2009. USPSTF Response to the ATT Collaboration Meta-Analysis. Agency for Healthcare Research and Quality, Rockville, Maryland.

http://www.ahrq.gov/clinic/tfcomments/tfaspattcom.htm

Cowen, T., 1988. The Theory of Market Failure: A Critical Examination. George Mason University Press, Fairfax, VA., ISBN: 0913969133.

Dominiczak, M.H., 2009. A matter of judgment: Clinical guidelines and scientific reductionism. Curr. Opin. Lipidol., 20: 446-447. DOI: 10.1097/MOL.0b013e328330998d

Hankin, R., 1996. Integrating Scientific Expertise into Regulatory Decision-Making: The Cases of Food and Pharmaceuticals. European University Institute, Florence, Italy. http://hdl.handle.net/1814/1422

Hedlund, J., 2000. Risky business: Safety regulations, risk compensation and individual behavior. Injury Prev., 6: 82-90. DOI: 10.1136/ip.6.2.82

Iizuka, T., 2006. Experts' Agency Problems: Evidence from the Prescription Drug Market in Japan. Unpublished Ph.D. Dissertation, University of California at Los Angeles, California. http://papers.ssrn.com/sol3/papers.cfm?abstract_id=949668

Kahn, R., R.M. Robertson, R. Smith and D. Eddy, 2008. The impact of prevention on reducing the burden of cardiovascular disease. Diabetes Care, 31: 1686-1696. DOI: 10.1161/CIRCULATIONAHA.108.190186

Keith, A., 1995. Regulating information about aspirin and the prevention of heart attack. Am. Econ. Rev., 85: 96-99.

Klein, D., 2000. Policy medicine versus policy quackery: Economists against the FDA. Knowl., Technol. Policy, 13: 92-101. DOI: 10.1007/s12130-000-1006-x

Lamotte, M., C. Piñol, C. Brotons, L. Annemans and E. Guardiola et al., 2006a. Health Economic evaluation of low-dose acetylsalicylic acid in the primary prevention of cardiovascular disease. Revista Espanola de Cardiologia, 59: 807-815. DOI: $10.1016 / \mathrm{S} 1885-5857(07) 60046-2$

Lamotte, M.A., L. Annemans, T. Evers and M. Kubin, 2006b. A multi-country economic evaluation of low-dose aspirin in the primary prevention of cardiovascular disease. PharmacoEconomics, 24: 155-169.

Mann, C.C. and M.L. Plummer, 1993. The Aspirin Wars: Money, Medicine and 100 Years of Rampant Competition. Harvard Business School Press, Boston, ISBN: 10: 0875844014.

Mathews, A.W., 2010. The danger of daily aspirin. Wall Street J., http://online.wsj.com/article/SB100014240527487 04511304575075701363436686.html
McKinlay J., C. Link, L. Marceau, A. O’Donnell and S. Arber et al., 2006. How do doctors in different countries manage the same patient? Results of a factorial experiment. Health Services Res., 41: 2182-2200. DOI: $10.1111 /$ j.14756773.2006.00595.x

Mendoza, R.L., 2008. Social welfare, public health policy and the theory of merit goods. J. Political Econ., 28: 57-65. http://www.freewebs.com/pennpgm/SOCWEL.pdf

Ogus, A., 2001. Regulatory Institutions and Structures (Paper No. 4). University of Manchester, Manchester, ISBN: 1-904056-03-2.

Pearson, D. and S. Shaw, 1994. Freedom of Informed Choice: FDA Versus Nutrient Supplements. Common Sense Press, Neptune, NJ., ISBN: 10: 0963624903.

Peltzman, S., 1973. The Benefits and Costs of New Drug Regulation. In: Regulating New Drugs, Landau, R.L. (Ed.). University of Chicago Press, Chicago, pp: 114-211.

Pildes, R.H. and C.R. Sunstein, 1995. Reinventing the regulatory state. Univ. Chicago Law Rev., 62: 1-129.

Pindyck, R.S. and D.L. Rubinfeld, 1989. Microeconomics. Macmillan Publishing Company, New York, ISBN: 0-02-395810-3.

Traynor, T.L., 2003. The impact of safety regulations on externalities. Atlant. Econ. J., 31: 62-70. DOI: 10.1007/BF02298463

Thun, M.J., S. J. Henley and C. Patrono, 2002. Nonsteroidal anti-inflammatory drugs as anticancer agents: Mechanistic, pharmacologic and clinical issues. J. Natl. Cancer Inst., 94: 252-266. DOI: 10.1093/jnci/94.4.252

United States Centers for Disease Control and Prevention, (CDC), 2010. FastStats. http://www.cdc.gov/nchs/fastats/lcod.htm

United States Preventive Services Task Force, (USPSTF), 2009. Aspirin for the prevention of cardiovascular disease. Ann. Inter. Med., 150: 396-404. http://www.annals.org/content/150/6/396.full

University of California at Berkeley, (UC Berkeley), 2006. Should Your Aspirin Wear a Coat? Wellness Letter, Berkeley, California. http://www.wellnessletter.com/html/wl/2006/wlFea tured1206.html

Wilde, G.J.S., 1994. Target Risk. PDE Publications, Toronto, ISBN: 0-9699124-0-4.

Wolff, T., T. Miller and S. Ko, 2009. Aspirin for the primary prevention of cardiovascular events: An update of the evidence for the U.S. preventive services task force. Ann. Inter. Med., 150: 405-410. http://www.annals.org/content/150/6/405.full.pdf+html 